\title{
Article \\ Incidence and Risk Factors for Progression to Diabetes Mellitus: A Retrospective Cohort Study
}

\author{
Min Kyung Hyun ${ }^{1}$, Jong Heon Park ${ }^{2}$, Kyoung Hoon Kim ${ }^{3}$, Soon-Ki Ahn ${ }^{4}(\mathbb{D})$ and Seon Mi Ji ${ }^{2, *}$ \\ 1 Department of Preventive Medicine, College of Korean Medicine, Dongguk University, Gyeongju 38066, \\ Korea; mk3three@dongguk.ac.kr \\ 2 National Health Insurance Service, Wonju 26464, Korea; parkjh@nhis.or.kr \\ 3 Health Insurance Review \& Assessment Service, Wonju 26465, Korea; rudgns112@hira.or.kr \\ 4 Public Health and Medical Services Office, Chungnam National University Hospital, Daejeon 35015, Korea; \\ withspirit09@gmail.com \\ * Correspondence: bleupink@nhis.or.kr; Tel.: +82-2-2126-8241
}

check for updates

Citation: Hyun, M.K.; Park, J.H.;

Kim, K.H.; Ahn, S.-K.; Ji, S.M.

Incidence and Risk Factors for

Progression to Diabetes Mellitus: A

Retrospective Cohort Study. Int. J.

Environ. Res. Public Health 2022, 19,

123. https://doi.org/10.3390/

ijerph19010123

Academic Editors: Rohini Mathur

and Sarah Cook

Received: 28 October 2021

Accepted: 16 December 2021

Published: 23 December 2021

Publisher's Note: MDPI stays neutral with regard to jurisdictional claims in published maps and institutional affiliations.

Copyright: () 2021 by the authors. Licensee MDPI, Basel, Switzerland. This article is an open access article distributed under the terms and conditions of the Creative Commons Attribution (CC BY) license (https:// creativecommons.org/licenses/by/ $4.0 /)$.

\begin{abstract}
Objective: This study examined the incidence and risk factors contributing to the progression to diabetes mellitus (DM) in a seven-year follow-up study of non-diabetic National Health Examinees. (2) Methods: For this retrospective observational cohort study, we used two national representative databases: the National Health Screening (HEALS) database 2009 and the National Health Insurance Service (NHIS) database 2009-2015. The eligible subjects without DM with blood sugar levels of $<126 \mathrm{mg} / \mathrm{dL}$ were selected using the HEALS database. The subsequent follow-up and clinical outcomes were evaluated using the NHIS database. Cox proportional hazard regression was applied to examine the effects of the covariates on progression to diabetes. (3) Results: Among those who took part in the national health screening in 2009, 4,205,006 subjects who met the eligibility criteria were selected. Of these, 587,015 were diagnosed with DM during the follow-up by 2015. The incidence of progression from non-diabetes to DM was $14.0 \%$, whereas that from impaired fasting glucose (IFG) to DM was $21.9 \%$. Compared to the normal group, the newly diagnosed DM group was more likely to comprise older, female, currently smoking, and high-risk drinking participants and participants with IFG, hypertension, dyslipidemia, and metabolic syndrome. (4) Conclusions: This epidemiological study in the Republic of Korea found risk factors similar to those of other studies, but the incidence of progression to DM was 22.8 per 1000 person-years, which is higher than that previously reported. Hence, more care is needed to prevent DM.
\end{abstract}

Keywords: diabetes mellitus; impaired fasting glucose; incidence; risk factors

\section{Introduction}

Diabetes mellitus (DM) is one of the world's leading public health concerns; it has increased steadily in incidence over the past few decades and resulted in complications in multiple organ systems [1]. The number of people with DM has risen from 285 million in 2009 to 463 million in 2019 and is expected to increase to 578 million by 2030 [1].

Prediabetes is defined when the glucose levels do not meet the criteria for DM but are too high to be considered normal, including impaired fasting glucose (IFG) and impaired glucose tolerance (IGT) [2]. Among them, IFG is diagnosed when the blood glucose levels are between 100 and $125 \mathrm{mg} / \mathrm{dL}(5.6$ to $7.0 \mathrm{mmol} / \mathrm{L})$, and it is highly associated with the development of DM [2]. IFG increases the risk of progression to DM, and the annual rate of conversion to DM is 6-9\% [3-5]. The prevalence of IFG ranges from $1 \%$ to $30 \%$, depending on the country and its estimated standards [6-8]. According to the American Diabetes Association (ADA), the risk factors for DM are age, gender, obesity, family history, physical inactivity, hypertension, and prediabetes including IFG [3]. On the other hand, the incidence and risk factors for chronic diseases, such as DM, can also show interregional, ethnic, and racial differences [9]. Indeed, some studies reported that Asian 
people have a higher risk of developing diabetes than other ethnicities, as well as a higher incidence [10,11]. A Japanese epidemiological study reported that IFG increased the risk of diabetes 8.8-fold [12]. However, few epidemiological studies on Koreans derived from a representative large-scale database that complements the strengths and weaknesses of each dataset have been reported.

Knowledge of the exact incidence and prevalence of prediabetes and diabetes is essential for effectively managing diabetes care and health insurance finances. In particular, the incidence of progression and risk factors of progression from non-diabetes, including IFG, to DM need to be determined.

In the present study, the subjects screened for normal glucose and IFG in the 2009 National Health Screening (HEALS) were followed up for seven years using the National Health Insurance Service (NHIS) database to investigate the incidence and risk factors of DM.

\section{Materials and Methods}

\subsection{Study Design and Data Source}

This was a retrospective observational cohort study using two national representative databases: the HEALS and NHIS databases owned by the NHIS system, a single insurer in the Republic of Korea (ROK) (Figure 1) [13,14]. The NHIS data cover approximately $96.6 \%$ of the ROK population and include the demographic and medical treatment information of the participants [13]. In contrast, HEALS data represent a cohort of people who participated in national health screening programs provided by the NHIS. It contains information on the health problems and risk factors of examinees obtained through the national health screening programs [14]. The HEALS and NHIS databases are linked to personal identification numbers, but the data are anonymized and provided to a designated secure computer in a security room. These data can be used to identify the incidence of new-onset DM among non-diabetic individuals at a health screen event and discover the risk factors for new-onset DM in the ROK.

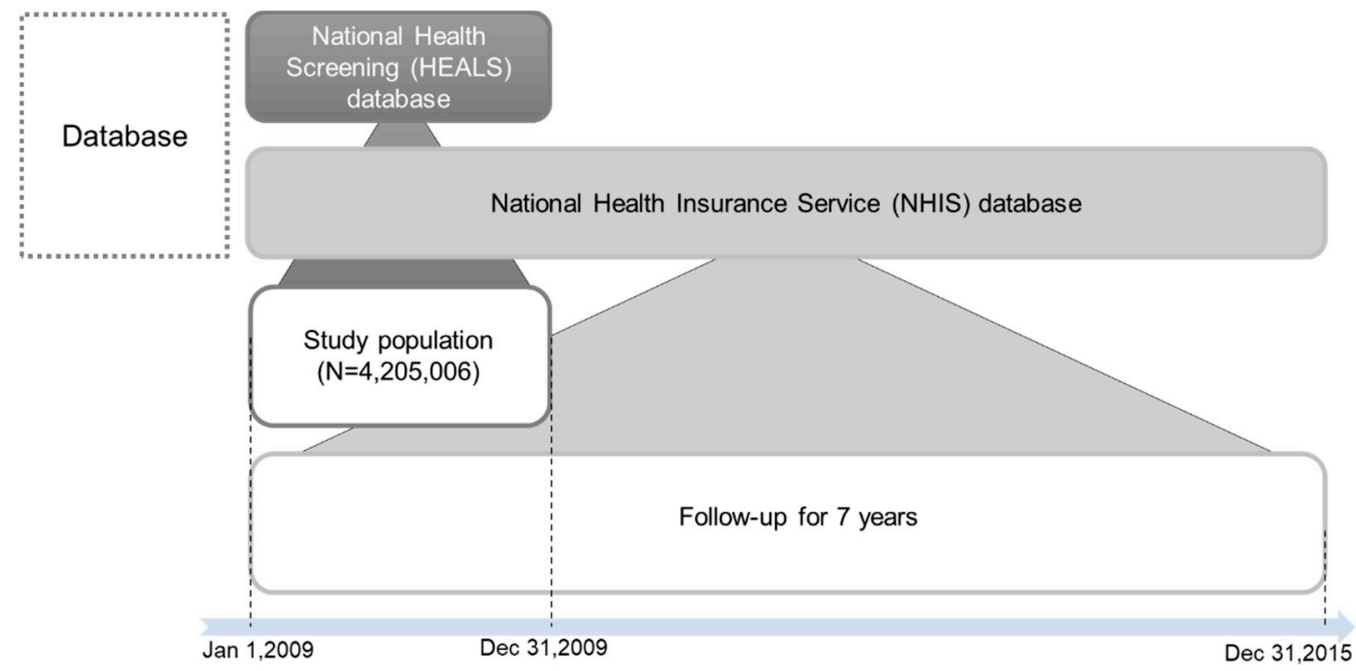

Figure 1. Study design.

\subsection{Study Subjects and Setting}

The eligible target subjects were selected using the HEALS database and subsequently followed up until 2015 when access was granted for analysis. The clinical outcomes were checked using the NHIS database (Figure 1).

Briefly, 10,644,911 adults participated in HEALS in 2009. The exclusion criteria were as follows: (i) initial blood sugar values of $\geq 126 \mathrm{mg} / \mathrm{dL}$, (ii) diagnosis of DM (ICD-10-CM: E10, 11, 13, and 14), (iii) history of malignancy (International Classification of Diseases, Tenth Revision, Clinical Modification (ICD-10-CM): C00 to 97), and (iv) missing data in 
the variables including smoking/drinking/exercise. Finally, 4,205,006 target subjects were selected for the study (Figure 2).

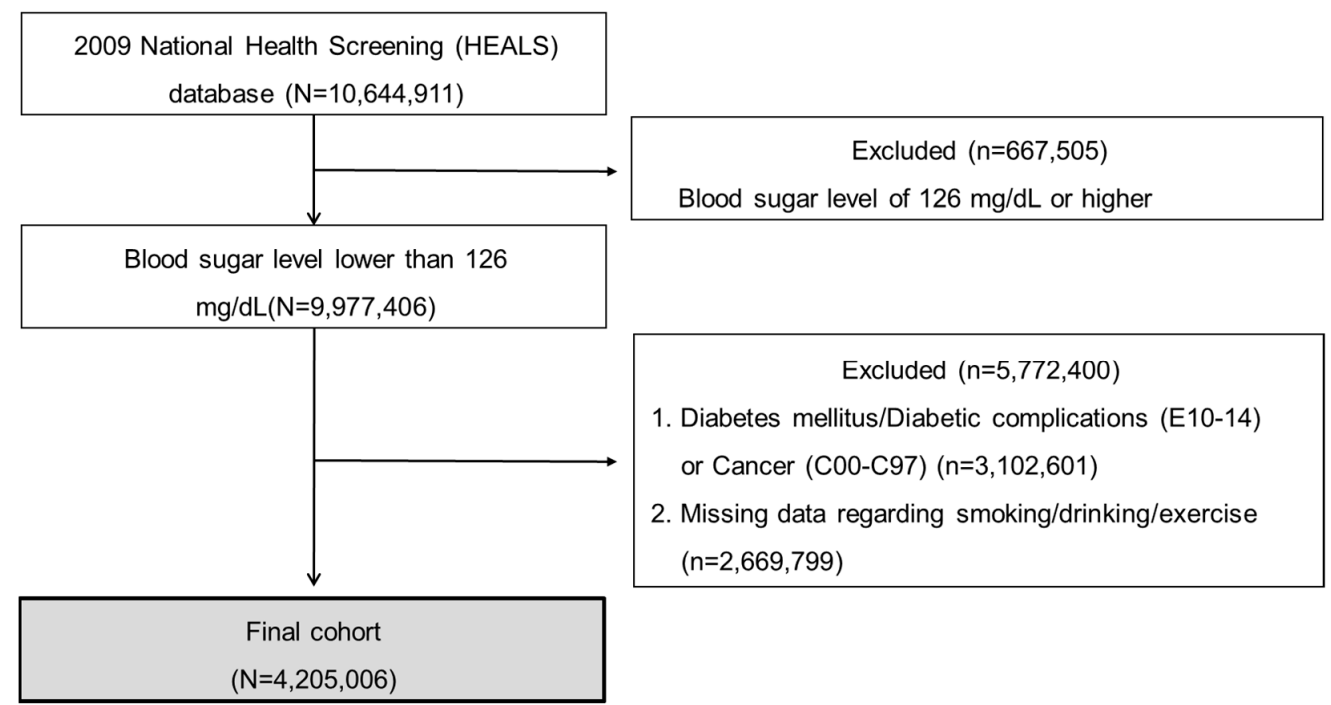

Figure 2. Selection of the study subjects.

\subsection{Variables}

\subsubsection{Outcome Variables}

The primary clinical endpoint was the progression from non-diabetes to DM, and new-onset DM was defined as a diagnosis with DM (ICD-10-CM: E10, 11, 13, 14) from the medical records of the NHIS database. The diagnostic criteria for DM in ROK are based on a fasting plasma glucose of $\geq 126 \mathrm{mg} / \mathrm{dL}$ for eight hours, or a two-hour plasma glucose of $\geq 200 \mathrm{mg} / \mathrm{dL}$ during a $75 \mathrm{~g}$ oral glucose tolerance test, or a glycosylated hemoglobin (A1C) level of $\geq 6.5 \%$ [15]. Repeated verification is required on another day if there were no obvious hyperglycemia symptoms (polyurea, polydipsia, and unexplained weight loss), but two or more of the above abnormal results from the same sample can be provided immediate confirmation [15].

\subsubsection{Household Income}

The household income was calculated based on the insurance owner's income level to claim health insurance premiums and was classified into quintiles. The higher the quintile, the higher the income level.

\subsubsection{Metabolic Syndrome}

Metabolic syndrome was defined in individuals meeting three or more of the following criteria: (1) abdominal obesity, with waist circumference of $\geq 90 \mathrm{~cm}$ in men or $\geq 85 \mathrm{~cm}$ in women; (2) hypertriglyceridemia, with triglyceride (TG) of $\geq 150 \mathrm{mg} / \mathrm{dL}$ or medication use; (3) low high-density lipoprotein (HDL)-cholesterol, with HDL-cholesterol of $<40 \mathrm{mg} / \mathrm{dL}$ in men and $<50 \mathrm{mg} / \mathrm{dL}$ in women; (4) high systolic blood pressure (BP), with systolic BP of $\geq 130 \mathrm{mmHg}$ and/or diastolic BP of $\geq 85 \mathrm{mmHg}$; or (5) hyperglycemia, with Fasting Plasma Glucose (FPG) of $>100 \mathrm{mg} / \mathrm{dL}$ or medication use [16].

\subsubsection{Current Smoking, High-Risk Drinking, and Proper Exercise}

Current smoking was defined in those who had smoked more than 100 cigarettes in their lives or were currently smoking [17].

High-risk drinking was defined as drinking more than $300 \mathrm{~mL}$ of alcoholic beverages per day on average. For traditional Korean drinks, one standard drink unit corresponds to one bowl (approximately $300 \mathrm{~mL}$ ) of Korean rice beer (Makgeoli) or a quarter bottle (approximately $90 \mathrm{~mL}$ ) of 20\% Korean liquor (Soju) [17,18]. 
Proper exercise was defined as follows: (1) intensive exercise lasting more than 20 min per session and more than three times per week or (2) moderate exercise lasting more than 30 min per session and more than five times per week $[19,20]$.

\subsection{Statistical Analyses}

The continuous variables are presented as the mean and standard deviation (SD) and were compared using Student's t-test. The categorical variables are presented as a proportion and were compared using a chi-square test. The relationships between the dependent variable (progression to DM) and the various risk factors or independent variables were examined via the Cox proportional hazard model. Multicollinearity analysis with the variance inflation factor (VIF) was performed to identify the collinearity between the variables. Variables with VIF $>5$ were considered to show severe multicollinearity; there were no variables with VIF $>5$ in the model. The Cox proportional hazard model was applied to consider the timing of the event. The onset of DM was defined as an event and was censored when the follow-up was terminated or death occurred. In other words, the Cox proportional hazard regression estimated the prognostic influence of the non-diabetes status on the conversion to DM, while simultaneously controlling for the confounding effects of covariates. This model estimated the instantaneous relative risk of conversion to DM, averaged over the entire follow-up duration. The proportional hazard assumption was tested using the goodness-of-fit test, which compares the observed and expected risk probabilities. The adjusted hazard ratios (HRs) and 95\% CIs are reported. Subgroup analysis was conducted on participants over 40 years of age because the prevalence of DM in Koreans in 2016 exceeded 10\% for men in their 40s and women in their 50s. The data were analyzed using SAS statistical software, version 9.4, for Windows (SAS, Cary, NC, USA); two-sided probability values less than 0.05 were considered significant.

\section{Results}

\subsection{Baseline Demographics}

Of the 4,205,006 participants analyzed, the mean age was $40.1 \pm 12.2$ years, with $71.8 \%$ being male. This is because males are more likely to participate in the national health screening [21]. The proportion of participants with IFG with fasting blood sugar levels of $100-125 \mathrm{mg} / \mathrm{dL}$ was $24.4 \%$, of which $80.6 \%$ were male. Metabolic syndrome was detected in $60.9 \%$ of the total: $64.0 \%$ males and $36.0 \%$ females. The proportions of all variables were significantly higher in males than in females $(p<0.001)$ (Table 1$)$.

Table 1. Characteristics of the participants.

\begin{tabular}{|c|c|c|c|c|c|c|c|c|}
\hline \multirow{2}{*}{\multicolumn{2}{|c|}{ Variables }} & \multicolumn{2}{|c|}{ Total } & \multicolumn{2}{|c|}{ Male } & \multicolumn{2}{|c|}{ Female } & \multirow{3}{*}{$p$-Value } \\
\hline & & \multirow{2}{*}{$\begin{array}{c}n \\
4,205,006\end{array}$} & \multirow{2}{*}{$\begin{array}{c}\% \\
100.0\end{array}$} & \multirow{2}{*}{$\begin{array}{c}n \\
3,019,103\end{array}$} & \multirow{2}{*}{$\begin{array}{c}\% \\
71.8 \%\end{array}$} & \multirow{2}{*}{$\begin{array}{c}n \\
1,185,903\end{array}$} & \multirow{2}{*}{$\begin{array}{c}\% \\
28.2 \%\end{array}$} & \\
\hline Total & & & & & & & & \\
\hline \multirow[t]{5}{*}{ IFG } & No & $3,179,247$ & $75.6 \%$ & $2,192,290$ & $69.0 \%$ & 986,957 & $31.0 \%$ & $<0.0001$ \\
\hline & Yes & $1,025,759$ & $24.4 \%$ & 826,813 & $80.6 \%$ & 198,946 & $19.4 \%$ & \\
\hline & & $40.1 \pm 12.2$ & & $40.4 \pm 11.9$ & & $39.3 \pm 12.8$ & & $<0.0001$ \\
\hline & $18 \sim 29$ & 977,664 & $23.3 \%$ & 615,826 & $63.0 \%$ & 361,838 & $37.0 \%$ & $<0.0001$ \\
\hline & $30 \sim 39$ & $1,254,309$ & $29.8 \%$ & 999,030 & $79.6 \%$ & 255,279 & $20.4 \%$ & \\
\hline \multirow[t]{4}{*}{ Age group } & $40 \sim 49$ & $1,078,746$ & $25.7 \%$ & 754,509 & $69.9 \%$ & 324,237 & $30.1 \%$ & \\
\hline & $50 \sim 59$ & 585,787 & $13.9 \%$ & 421,115 & $71.9 \%$ & 164,672 & $28.1 \%$ & \\
\hline & $60 \sim 69$ & 237,616 & $5.7 \%$ & 177,846 & $74.8 \%$ & 59,770 & $25.2 \%$ & \\
\hline & $\geq 70$ & 70,884 & $1.7 \%$ & 50,777 & 1.68 & 20,107 & $28.4 \%$ & \\
\hline \multirow[t]{3}{*}{ Household income } & 1 & 647,627 & $15.4 \%$ & 400,233 & $61.8 \%$ & 247,394 & $38.2 \%$ & $<0.0001$ \\
\hline & 2 & 747,199 & $17.8 \%$ & 455,816 & $61.0 \%$ & 291,383 & $39.0 \%$ & \\
\hline & 3 & 928,682 & $22.1 \%$ & 669,523 & $72.1 \%$ & 259,159 & $27.9 \%$ & \\
\hline
\end{tabular}


Table 1. Cont.

\begin{tabular}{|c|c|c|c|c|c|c|c|c|}
\hline \multirow{2}{*}{\multicolumn{2}{|c|}{ Variables }} & \multicolumn{2}{|c|}{ Total } & \multicolumn{2}{|c|}{ Male } & \multicolumn{2}{|c|}{ Female } & \multirow{3}{*}{$p$-Value } \\
\hline & & $n$ & $\%$ & $n$ & $\%$ & $n$ & $\%$ & \\
\hline \multicolumn{2}{|c|}{ Total (n) } & $4,205,006$ & 100.0 & $3,019,103$ & $71.8 \%$ & $1,185,903$ & $28.2 \%$ & \\
\hline \multirow{4}{*}{ Hypertension } & 4 & 989,910 & $23.5 \%$ & 775,989 & $78.4 \%$ & 213,921 & $21.6 \%$ & \\
\hline & 5 & 891,588 & $21.2 \%$ & 717,542 & $80.5 \%$ & 174,046 & $19.5 \%$ & \\
\hline & No & $3,901,156$ & $92.8 \%$ & $2,784,454$ & $71.4 \%$ & $1,116,702$ & $28.6 \%$ & $<0.0001$ \\
\hline & Yes & 303,850 & $7.2 \%$ & 234,649 & $77.2 \%$ & 69,201 & $22.8 \%$ & \\
\hline \multirow{3}{*}{$\begin{array}{l}\text { Systolic blood } \\
\text { pressure }\end{array}$} & $<120$ & $1,720,188$ & $40.9 \%$ & $1,012,586$ & $58.9 \%$ & 707,602 & $41.1 \%$ & $<0.0001$ \\
\hline & $120 \sim 139$ & $2,083,991$ & $49.6 \%$ & $1,673,166$ & $80.3 \%$ & 410,825 & $19.7 \%$ & \\
\hline & $\geq 140$ & 400,827 & $9.5 \%$ & 333,351 & $83.2 \%$ & 67,476 & $16.8 \%$ & \\
\hline \multirow{3}{*}{$\begin{array}{c}\text { Diastolic blood } \\
\text { pressure }\end{array}$} & $<80$ & $2,279,381$ & $54.2 \%$ & $1,441,408$ & $63.2 \%$ & 837,973 & $36.8 \%$ & $<0.0001$ \\
\hline & $80 \sim 89$ & $1,522,799$ & $36.2 \%$ & $1,236,603$ & $81.2 \%$ & 286,196 & $18.8 \%$ & \\
\hline & $\geq 90$ & 402,826 & $9.6 \%$ & 341,092 & $84.7 \%$ & 61,734 & $15.3 \%$ & \\
\hline \multirow{2}{*}{ Dyslipidemia } & $\overline{\mathrm{No}}$ & $4,108,644$ & $97.7 \%$ & $2,939,918$ & $71.6 \%$ & $1,168,726$ & $28.4 \%$ & $<0.0001$ \\
\hline & Yes & 96,362 & $2.3 \%$ & 79,185 & $82.2 \%$ & 17,177 & $17.8 \%$ & \\
\hline \multirow{2}{*}{ Triglyceride } & $<150$ & $2,926,610$ & $69.6 \%$ & $1,887,318$ & $64.5 \%$ & $1,039,292$ & $35.5 \%$ & $<0.0001$ \\
\hline & $\geq 150$ & $1,278,396$ & $30.4 \%$ & $1,131,785$ & $88.5 \%$ & 146,611 & $11.5 \%$ & \\
\hline \multirow{2}{*}{ HDL } & $\mathrm{M}<\overline{40}, \mathrm{~F}<50$ & 594,305 & $14.1 \%$ & 362,835 & $61.1 \%$ & 231,470 & $38.9 \%$ & $<0.0001$ \\
\hline & $\mathrm{M} \geq 40, \mathrm{~F} \geq 50$ & $3,610,701$ & $85.9 \%$ & $2,656,268$ & $73.6 \%$ & 954,433 & $26.4 \%$ & \\
\hline BMI & & $23.6 \pm 3.2$ & & $24.1 \pm 3.0$ & & $22.4 \pm 3.2$ & & $<0.0001$ \\
\hline Waist & $\mathrm{M}<90, \mathrm{~F}<85$ & $3,524,867$ & $83.8 \%$ & 2,461,292 & $69.8 \%$ & $1,063,575$ & $30.2 \%$ & $<0.0001$ \\
\hline circumference & $\mathrm{M} \geq 90, \mathrm{~F} \geq 85$ & 680,139 & $16.2 \%$ & 557,811 & $82.0 \%$ & 122,328 & $18.0 \%$ & \\
\hline Metabolic & No & $1,643,100$ & $39.1 \%$ & $1,380,427$ & $84.0 \%$ & 262,673 & $16.0 \%$ & $<0.0001$ \\
\hline syndrome & Yes & $2,561,906$ & $60.9 \%$ & $1,638,676$ & $64.0 \%$ & 923,230 & $36.0 \%$ & \\
\hline \multirow{2}{*}{ Current smoking } & No & $2,540,898$ & $60.4 \%$ & $1,427,760$ & $56.2 \%$ & $1,113,138$ & $43.8 \%$ & $<0.0001$ \\
\hline & Yes & $1,664,108$ & $39.6 \%$ & $1,591,343$ & $95.6 \%$ & 72,765 & $4.4 \%$ & \\
\hline \multirow{2}{*}{ High-risk drinking } & No & $3,645,544$ & $86.7 \%$ & $2,495,166$ & $68.4 \%$ & $1,150,378$ & $31.6 \%$ & $<0.0001$ \\
\hline & Yes & 559,462 & $13.3 \%$ & 523,937 & $93.7 \%$ & 35,525 & $6.3 \%$ & \\
\hline \multirow{2}{*}{ Proper exercise } & No & $3,452,613$ & $82.1 \%$ & $2,436,072$ & $70.6 \%$ & $1,016,541$ & $29.4 \%$ & $<0.0001$ \\
\hline & Yes & 752,393 & $17.9 \%$ & 583,031 & $77.5 \%$ & 169,362 & $22.5 \%$ & \\
\hline
\end{tabular}

Abbreviations: IFG, impaired fasting glucose; HDL, high-density lipoprotein; BMI, body mass index.

\subsection{Incidence and Characteristics of Progression from Non-Diabetes to Diabetes}

The cumulative incidence of DM was 14\% for the seven-year follow-up, and the conversion rate of non-diabetes to DM was 22.8 per 1000 person-years (Table 2). The mean age of the 587,015 new-onset DM cases was $48.0 \pm 12.7$ years, which was higher than the $38.8 \pm 11.6$ years in the patients not diagnosed with DM; $21.9 \%$ of IFG subjects were diagnosed with DM compared to $11.4 \%$ of subjects with normal blood sugar levels; and $33.7 \%$ of subjects with hypertension were diagnosed with DM, which was higher than the $12.4 \%$ of non-hypertensive cases (Table 3 ).

Table 2. Incidence of progression from non-diabetes to diabetes.

\begin{tabular}{|c|c|c|c|c|c|c|c|}
\hline $\begin{array}{l}\text { Follow-Up } \\
\text { Period }\end{array}$ & $\begin{array}{l}\text { Follow-Up } \\
\text { Population }\end{array}$ & $\begin{array}{c}\text { Newly Diagnosed } \\
\text { Diabetes }\end{array}$ & Censored $^{1}$ & $\begin{array}{c}\text { Cumulative Incidence } \\
\text { of Diabetes }\end{array}$ & & Person-Years ${ }^{2}$ & Conversion \\
\hline Year & $\mathbf{N}$ & $n$ & $n$ & $n$ & $\%$ & & \\
\hline$<1$ & $4,205,006$ & 55,129 & 4,165 & 55,129 & $1.3 \%$ & $4,202,924$ & 13.1 \\
\hline $1 \sim 2$ & $4,145,712$ & 88,029 & 4,881 & 143,158 & $3.4 \%$ & $4,143,272$ & 21.2 \\
\hline $2 \sim 3$ & $4,052,802$ & 93,463 & 5,259 & 236,621 & $5.6 \%$ & $4,050,173$ & 23.1 \\
\hline $3 \sim 4$ & $3,954,080$ & 95,179 & 5,286 & 331,800 & $7.9 \%$ & $3,951,437$ & 24.1 \\
\hline $4 \sim 5$ & $3,853,615$ & 102,731 & 4,910 & 434,531 & $10.3 \%$ & $3,851,160$ & 26.7 \\
\hline $5 \sim 6$ & $3,745,974$ & 104,789 & 4,321 & 539,320 & $12.8 \%$ & $3,743,814$ & 28.0 \\
\hline $6 \sim 7$ & $3,636,864$ & 47,695 & $3,589,169$ & 587,015 & $14.0 \%$ & $1,842,280$ & 25.9 \\
\hline Total & $4,205,006$ & & & 587,015 & $14.0 \%$ & $25,785,058$ & 22.8 \\
\hline
\end{tabular}

${ }^{1}$ If the follow-up was terminated or death occurred; ${ }^{2}$ Censored times half-year assumption (follow-up population $-0.5 \times$ Censored); ${ }^{3}$ Number of diabetes progressions from non-diabetes per 1000 person-years. 
Table 3. Characteristics of the progression from non-diabetes to diabetes.

\begin{tabular}{|c|c|c|c|c|c|c|}
\hline \multirow{2}{*}{\multicolumn{2}{|c|}{ Variables }} & \multicolumn{2}{|c|}{ Diabetes Diagnosis } & \multicolumn{2}{|c|}{ No Diabetes Diagnosis } & \multirow{3}{*}{$p$-Value } \\
\hline & & \multirow{2}{*}{$\begin{array}{c}n \\
587,015\end{array}$} & \multirow{2}{*}{$\begin{array}{c}\% \\
(14.0)\end{array}$} & \multirow{2}{*}{$\begin{array}{c}n \\
3,617,991\end{array}$} & \multirow{2}{*}{$\begin{array}{c}\% \\
(86.0)\end{array}$} & \\
\hline Total & & & & & & \\
\hline \multirow{2}{*}{ IFG } & No & 362,128 & (11.4) & $2,817,119$ & $(88.6)$ & $<0.0001$ \\
\hline & Yes & 224,887 & (21.9) & 800,872 & $(78.1)$ & \\
\hline \multirow{7}{*}{ Age group } & & $48.0 \pm 12.7$ & & $38.8 \pm 11.6$ & & $<0.0001$ \\
\hline & $18-29$ & 49,669 & $(5.1)$ & 927,995 & $(94.9)$ & \\
\hline & $30-39$ & 107,231 & $(8.6)$ & $1,147,078$ & (91.5) & \\
\hline & $40-49$ & 169,599 & (15.7) & 909,147 & (84.3) & \\
\hline & $50-59$ & 150,062 & $(25.6)$ & 435,725 & $(74.4)$ & \\
\hline & $60-69$ & 82,528 & $(34.7)$ & 155,088 & $(65.3)$ & \\
\hline & $\geq 70$ & 27,926 & $(39.4)$ & 42,958 & $(60.6)$ & \\
\hline \multirow{2}{*}{ Gender } & Male & 426,674 & (14.1) & $2,592,429$ & $(85.9)$ & $<0.0001$ \\
\hline & Female & 160,341 & (13.5) & $1,025,562$ & $(86.5)$ & \\
\hline \multirow{5}{*}{ Household income } & 1 & 96,040 & (14.8) & 551,587 & $(85.2)$ & $<0.0001$ \\
\hline & 2 & 94,698 & $(12.7)$ & 652,501 & $(87.3)$ & \\
\hline & 3 & 116,804 & (12.6) & 811,878 & $(87.4)$ & \\
\hline & 4 & 136,771 & (13.8) & 853,139 & $(86.2)$ & \\
\hline & 5 & 142,702 & $(16.0)$ & 748,886 & $(84.0)$ & \\
\hline \multirow{2}{*}{ Hypertension } & No & 484,769 & (12.4) & $3,416,387$ & $(87.6)$ & $<0.0001$ \\
\hline & Yes & 102,246 & (33.7) & 201,604 & $(66.4)$ & \\
\hline \multirow{4}{*}{ Systolic blood pressure } & $<120$ & 182,114 & (10.6) & $1,538,074$ & $(89.4)$ & $<0.0001$ \\
\hline & $120-139$ & 304,390 & $(14.6)$ & $1,779,601$ & $(85.4)$ & \\
\hline & $\geq 140$ & 100,511 & $(25.1)$ & 300,316 & $(74.9)$ & \\
\hline & $<80$ & 264,121 & (11.6) & $2,015,260$ & $(88.4)$ & $<0.0001$ \\
\hline \multirow[t]{2}{*}{ Diastolic blood pressure } & $80-89$ & 230,515 & $(15.1)$ & $1,292,284$ & $(84.9)$ & \\
\hline & $\geq 90$ & 92,379 & $(22.9)$ & 310,447 & $(77.1)$ & \\
\hline \multirow{2}{*}{ Dyslipidemia } & $\overline{\mathrm{No}}$ & 562,230 & (13.7) & $3,546,414$ & $(86.3)$ & $<0.0001$ \\
\hline & Yes & 24,785 & $(25.7)$ & 71,577 & $(74.3)$ & \\
\hline \multirow{2}{*}{ Triglyceride } & $<150$ & 354,226 & $(12.1)$ & $2,572,384$ & (87.9) & $<0.0001$ \\
\hline & $\geq 150$ & 232,789 & (18.2) & $1,045,607$ & $(81.8)$ & \\
\hline \multirow{2}{*}{ HDL } & $\mathrm{M}<40, \mathrm{~F}<50$ & 103,175 & $(17.4)$ & 491,130 & $(82.6)$ & $<0.0001$ \\
\hline & $\mathrm{M} \geq 40, \mathrm{~F} \geq 50$ & 483,840 & (13.4) & $3,126,861$ & $(86.6)$ & \\
\hline BMI & & $24.5 \pm 3.3$ & & $23.4 \pm 3.1$ & & $<0.0001$ \\
\hline \multirow{2}{*}{ Waist circumference } & $\mathrm{M}<90, \mathrm{~F}<85$ & 433,978 & $(12.3)$ & $3,090,889$ & $(87.7)$ & $<0.0001$ \\
\hline & $\mathrm{M} \geq 90, \mathrm{~F} \geq 85$ & 153,037 & $(22.5)$ & 527,102 & $(77.5)$ & \\
\hline \multirow{2}{*}{ Metabolic syndrome } & No & 300,847 & (18.3) & $1,342,253$ & $(81.7)$ & $<0.0001$ \\
\hline & Yes & 286,168 & (11.2) & $2,275,738$ & $(88.8)$ & \\
\hline \multirow{2}{*}{ Current smoking } & No & 367,954 & $(14.5)$ & $2,172,944$ & $(85.5)$ & $<0.0001$ \\
\hline & Yes & 219,061 & $(13.2)$ & $1,445,047$ & $(86.8)$ & \\
\hline \multirow{2}{*}{ High-risk drinking } & No & 492,266 & (13.5) & $3,153,278$ & (86.5) & $<0.0001$ \\
\hline & Yes & 94,749 & $(16.9)$ & 464,713 & $(83.1)$ & \\
\hline \multirow{2}{*}{ Proper exercise } & No & 468,582 & (13.6) & $2,984,031$ & $(86.4)$ & $<0.0001$ \\
\hline & Yes & 118,433 & $(15.7)$ & 633,960 & $(84.3)$ & \\
\hline
\end{tabular}

\subsection{Risk Factors of Progression from Non-Diabetes to Diabetes}

The newly diagnosed DM group were characterized by IFG (HR 1.552, 95\% CI: 1.542 1.563), older age (if 70 and above, HR 7.504, 95\% CI: 7.389-7.620), female sex (HR 1.198, 95\% CI: 1.190-1.206), high household income (if fifth quintile, HR 0.931, 95\% CI: 0.924-0.939), hypertension (HR 1.433, 95\% CI: 1.433-1.454), dyslipidemia (HR 1.256, 95\% CI: 1.239-1.272), high triglyceride (HR 1.203, 95\% CI: 1.195-1.212), high BMI (HR 1.048, 95\% CI: 1.046-1.049), metabolic syndrome (HR 1040, 95\% CI: 1.030-1.049), current smoking (HR 1082, 95\% CI: 1.075-1.088), and high-risk drinking (HR 1140, 95\% CI: 1.132-1.148), as compared to the normal group (Table 4). The results were similar in the analysis of participants aged 40 years and over (Table 5). 
Table 4. Risk factors for the progression from non-diabetes to diabetes.

\begin{tabular}{|c|c|c|c|c|c|}
\hline \multicolumn{2}{|c|}{ Variables } & \multirow{2}{*}{$\begin{array}{c}\text { Hazard }{ }^{*} \text { Ratio } \\
1.000\end{array}$} & \multirow[t]{2}{*}{ 95\% Lower CI } & \multirow[t]{2}{*}{$95 \%$ Upper CI } & \multirow[t]{2}{*}{$p$-Value } \\
\hline \multirow{3}{*}{ IFG } & No & & & & \\
\hline & Yes & 1.552 & 1.542 & 1.563 & $<0.0001$ \\
\hline & $18-29$ & 1.000 & & & \\
\hline \multirow{4}{*}{ Age group } & $30-39$ & 1.509 & 1.492 & 1.525 & $<0.0001$ \\
\hline & $40-49$ & 2.724 & 2.696 & 2.752 & $<0.0001$ \\
\hline & $50-59$ & 4.382 & 4.336 & 4.429 & $<0.0001$ \\
\hline & $60-69$ & 6.047 & 5.977 & 6.119 & $<0.0001$ \\
\hline \multirow{4}{*}{ Gender } & $\geq 70$ & 7.504 & 7.389 & 7.620 & $<0.0001$ \\
\hline & Male & 1.000 & & & \\
\hline & Female & 1.198 & 1.190 & 1.206 & $<0.0001$ \\
\hline & 1 & 1.000 & & & \\
\hline \multirow{3}{*}{ Household income } & 2 & 1.003 & 0.994 & 1.012 & 0.5275 \\
\hline & 3 & 1.005 & 0.997 & 1.014 & 0.2438 \\
\hline & 4 & 1.008 & 0.999 & 1.016 & 0.0732 \\
\hline & 5 & 0.931 & 0.924 & 0.939 & $<0.0001$ \\
\hline \multirow[t]{3}{*}{ Hypertension } & No & 1.000 & & & \\
\hline & Yes & 1.443 & 1.433 & 1.454 & $<0.0001$ \\
\hline & $<120$ & 1.000 & & & \\
\hline \multirow[t]{3}{*}{ Systolic blood pressure } & $120-139$ & 1.051 & 1.043 & 1.059 & $<0.0001$ \\
\hline & $\geq 140$ & 1.176 & 1.162 & 1.189 & $<0.0001$ \\
\hline & $<80$ & 1.000 & & & \\
\hline \multirow[t]{2}{*}{ Diastolic blood pressure } & $80-89$ & 1.023 & 1.016 & 1.030 & $<0.0001$ \\
\hline & $\geq 90$ & 1.082 & 1.070 & 1.094 & $<0.0001$ \\
\hline \multirow{2}{*}{ Dyslipidemia } & No & 1.000 & & & \\
\hline & Yes & 1.256 & 1.239 & 1.272 & $<0.0001$ \\
\hline \multirow{2}{*}{ Triglyceride } & $<150$ & 1.000 & & & \\
\hline & $\geq 150$ & 1.203 & 1.195 & 1.212 & $<0.0001$ \\
\hline \multirow{2}{*}{ HDL } & $\mathrm{M}<\overline{40}, \mathrm{~F}<50$ & 1.000 & & & \\
\hline & $\mathrm{M} \geq 40, \mathrm{~F} \geq 50$ & 0.937 & 0.930 & 0.945 & $<0.0001$ \\
\hline BMI & & 1.048 & 1.046 & 1.049 & $<0.0001$ \\
\hline \multirow[b]{2}{*}{ Waist circumference } & $\mathrm{M}<90, \mathrm{~F}<85$ & 1.000 & & & \\
\hline & $M \geq 90, F \geq 85$ & 1.183 & 1.173 & 1.193 & $<0.0001$ \\
\hline \multirow{2}{*}{ Metabolic syndrome } & No & 1.000 & & & \\
\hline & Yes & 1.040 & 1.030 & 1.049 & $<0.0001$ \\
\hline \multirow{2}{*}{ Current smoking } & No & 1.000 & & & \\
\hline & Yes & 1.082 & 1.075 & 1.088 & $<0.0001$ \\
\hline \multirow{2}{*}{ High-risk drinking } & No & 1.000 & & & \\
\hline & Yes & 1.140 & 1.132 & 1.148 & $<0.0001$ \\
\hline \multirow{2}{*}{ Proper exercise } & No & 1.000 & & & \\
\hline & Yes & 0.999 & 0.993 & 1.005 & 0.7664 \\
\hline
\end{tabular}

* Adjusted for IFG, age group, gender, household income, hypertension, systolic blood pressure, diastolic blood pressure, dyslipidemia, triglyceride, HDL, BMI, waist circumference, metabolic syndrome, current smoking, high-risk drinking, and proper exercise.

Table 5. Risk factors for the progression from non-diabetes to diabetes from age 40.

\begin{tabular}{|c|c|c|c|c|c|}
\hline \multicolumn{2}{|c|}{ Variables } & \multirow{2}{*}{$\frac{\text { Hazard }{ }^{*} \text { Ratio }}{1.000}$} & \multirow[t]{2}{*}{ 95\% Lower CI } & \multirow[t]{2}{*}{$95 \%$ Upper CI } & \multirow[t]{2}{*}{$p$-Value } \\
\hline & No & & & & \\
\hline IFG & Yes & 1.521 & 1.509 & 1.533 & $<0.0001$ \\
\hline Age & & 1.040 & 1.039 & 1.040 & $<0.0001$ \\
\hline \multirow{4}{*}{ Gender } & Male & 1.000 & & & \\
\hline & Female & 1.145 & 1.137 & 1.154 & $<0.0001$ \\
\hline & 1 & 1.000 & & & \\
\hline & 2 & 1.001 & 0.990 & 1.011 & 0.8834 \\
\hline \multirow{3}{*}{ Household income } & 3 & 1.000 & 0.990 & 1.011 & 0.9368 \\
\hline & 4 & 0.998 & 0.989 & 1.008 & 0.718 \\
\hline & 5 & 0.908 & 0.900 & 0.917 & $<0.0001$ \\
\hline
\end{tabular}


Table 5. Cont.

\begin{tabular}{|c|c|c|c|c|c|}
\hline \multicolumn{2}{|c|}{ Variables } & \multirow{2}{*}{$\begin{array}{c}\text { Hazard }{ }^{*} \text { Ratio } \\
1.000\end{array}$} & \multirow[t]{2}{*}{ 95\% Lower CI } & \multirow[t]{2}{*}{$95 \%$ Upper CI } & \multirow[t]{2}{*}{$p$-Value } \\
\hline \multirow{3}{*}{ Hypertension } & No & & & & \\
\hline & Yes & 1.394 & 1.383 & 1.405 & $<0.0001$ \\
\hline & $<120$ & 1.000 & & & \\
\hline \multirow[t]{3}{*}{ Systolic blood pressure } & $120-139$ & 1.051 & 1.042 & 1.060 & $<0.0001$ \\
\hline & $\geq 140$ & 1.134 & 1.120 & 1.149 & $<0.0001$ \\
\hline & $<80$ & 1.000 & & & \\
\hline \multirow[t]{2}{*}{ Diastolic blood pressure } & $80-89$ & 1.021 & 1.013 & 1.029 & $<0.0001$ \\
\hline & $\geq 90$ & 1.068 & 1.054 & 1.081 & $<0.0001$ \\
\hline \multirow{2}{*}{ Dyslipidemia } & No & 1.000 & & & \\
\hline & Yes & 1.248 & 1.231 & 1.266 & $<0.0001$ \\
\hline \multirow{2}{*}{ Triglyceride } & $<150$ & 1.000 & & & \\
\hline & $\geq 150$ & 1.176 & 1.167 & 1.186 & $<0.0001$ \\
\hline \multirow{2}{*}{ HDL } & $\mathrm{M}<40, \mathrm{~F}<50$ & 1.000 & & & \\
\hline & $\mathrm{M} \geq 40, \mathrm{~F} \geq 50$ & 0.943 & 0.935 & 0.952 & $<0.0001$ \\
\hline BMI & & 1.042 & 1.041 & 1.043 & $<0.0001$ \\
\hline \multirow{2}{*}{ Waist circumference } & $\mathrm{M}<90, \mathrm{~F}<85$ & 1.000 & & & \\
\hline & $\mathrm{M} \geq 90, \mathrm{~F} \geq 85$ & 1.145 & 1.135 & 1.156 & $<0.0001$ \\
\hline \multirow{2}{*}{ Metabolic syndrome } & No & 1.000 & & & \\
\hline & Yes & 1.023 & 1.013 & 1.034 & $<0.0001$ \\
\hline \multirow{2}{*}{ Current smoking } & No & 1.000 & & & \\
\hline & Yes & 1.103 & 1.096 & 1.111 & $<0.0001$ \\
\hline \multirow{2}{*}{ High-risk drinking } & No & 1.000 & & & \\
\hline & Yes & 1.131 & 1.122 & 1.141 & $<0.0001$ \\
\hline \multirow{2}{*}{ Proper exercise } & No & 1.000 & & & \\
\hline & Yes & 0.996 & 0.988 & 1.003 & 0.2366 \\
\hline
\end{tabular}

* Adjusted for IFG, age, gender, household income, hypertension, systolic blood pressure, diastolic blood pressure, dyslipidemia, triglyceride, HDL, BMI, waist circumference, metabolic syndrome, current smoking, high-risk drinking, and proper exercise.

\section{Discussion}

The incidence of and risk factors contributing to DM progression in a follow-up study of non-diabetic national health examinees were examined by linking two types of national representative databases in the ROK. Approximately $14 \%$ of people not diagnosed with DM in the 2009 National Health Screening were diagnosed with DM over the seven-year follow-up. Furthermore, $24.4 \%$ of all subjects had IFG at the 2009 screening; $21.9 \%$ of those had converted to DM by 2015. In addition, the conversion rate from non-diabetes to DM was 22.8 per 1000 person-years.

A study of the 40 69-year age group without DM at the baseline examination in 2001 2002 using the Korean Genome and Epidemiology Study (KoGES) revealed an overall DM incidence of 22.1 per 1000 person-years after a 12-year follow-up [22]. The follow-up period was longer than that of the present study, and the study subjects were limited to residents of certain regions over 40 years of age. On the other hand, the conversion rate was 22.1, which is slightly lower than that in the present findings. These results may be because the data was collected in 2001-much earlier than 2009, the collection year of the present study. According to the NHIS database, the number of DM patients has increased steadily from 2.7 million in 2016 to 3.3 million in 2020 . Of these, $95 \%$ of patients with DM in 2020 were in their 40 s or older [23].

According to a previous study, among the 6.4 million members of the Hong Kong population who used hospital authority services from 2006 to 2014, the incidence of DM was $5.9 \%(n=377,565)$, and the conversion rate to DM was 9.46 per 1000 person-years in 2014, which is much lower than that in the present findings [24]. In relatively old data from Japan, a systematic review and meta-analysis of the studies conducted between 1980 and 2003 resulted in a pooled DM incidence rate of 8.8 (95\% confidence interval, 7.4-10.4) per 1000 person-years [25]. On the other hand, the Chennai Urban Rural Epidemiology Study (CURES) cohort $(n=1376)$, which followed an Asian Indian cohort for 9.1 years until 2013, 
reported a 30\% incidence of DM [10]. In addition, they reported DM conversion rates of 33.1 per 1000 person-years in non-diabetics, including those with prediabetes, and 61.0 per 1000 person-years in IFG subjects [10]. In this study, female subjects exhibited 1.01 times higher conversion from non-diabetes to DM and 1.10 times higher conversion from IFG/IGT to DM than male subjects, but this difference was not statistically significant [10]. Similarily, the conversion to DM was 1.521 times higher in IFG subjects than in normal-glucose subjects, and the conversion to DM in non-diabetics was 1.198 times higher in females than in males [10]. On the other hand, the incidence of DM and the conversion rate of DM were overwhelmingly higher than those of the present research. At the start of the observation, the proportion of IFG subjects was $4.9 \%$ (while the proportion of all types of prediabetic subjects was $21.7 \%$ ), which is lower than the $24.4 \%$ of IFG in the present study [10]. These differences may be related to the metabolic effects of the western-style diet, or tissue resistance to insulin. One study reported that Asian Indian people have the highest incidence of DM when compared to other Asian people [26]. The Southall And Brent Revisited (SABRE) study $(n=1007)$ that observed South Asian men 40-69 years of age living in North and West London for 19 years until 2011 reported a 35\% incidence of DM [11]. This is higher than the $14.1 \%$ incidence of DM in men in the present study. Therefore, Asian ethnic groups have different incidence rates of DM, depending on their growth environment.Accordingly, more epidemiological studies are needed under a range of conditions to better understand the status and trends, suppress the increase in the incidence of prediabetes and DM, and set healthcare policies for prevention and treatment that are compatible with the burden of prediabetes and DM.

In the present study and reported research, IFG is a risk factor of progression to DM. A systematic review by the U.S. Preventive Services Task Force (USPSTF) reported that the treatment of IFG is associated with delayed progression to DM [27]. Therefore, it is necessary to prevent progression to DM in subjects with IFG by applying the appropriate lifestyle and medical interventions [2]. In particular, subjects with IFG are at higher risk of developing cardiovascular disease. Therefore, they require intensive cardiovascular risk management [28]. The National Institute for Health and Care Excellence (NICE) suggested a national strategy and policy to prevent DM linked to diet, physical activity, and obesity. In addition, adult DM patients need to manage their blood pressure, lipids and cardiovascular risk, blood glucose, and complications [29]. In line with these recommendations, hypertension, dyslipidemia, higher BMI, and metabolic syndrome were also risk factors for DM in the present study. However, there is also a report that IGT is not a risk factor for conversion to diabetes, which may explain partly why some subjects without metabolic syndrome converted to diabetes in present study [30].

Health behaviors are also linked to the development of DM. Smoking increases the risk of DM by affecting visceral abdominal fat accumulation, insulin resistance, and pancreatic b-cell dysfunction [31,32]. Various epidemiological studies have demonstrated a risk of DM and its complications linked to smoking [33-36]. Alcohol consumption also increases the risk of DM and its complications by affecting plasma glucose, gluconeogenesis, and insulin sensitivity [37]. In contrast, physical activity decreases the risk of DM and its complications [38-40]. In the present study, proper exercise was not a significant factor in the incidence of DM, which appears to be due to the limitation that it is not an accurate measurement of the amount and quality of exercise. Unlike smoking and drinking, exercise may have behavioral variations in terms of continuity and addictive behavior. Therefore, repeated measurement data using accurate tools will be needed. For example, a Korean study measured physical activity using a self-reported international physical activity questionnaire and reported a lower trend in the incidence of DM [38]. Unlike the incidence and conversion rates of DM, racial and regional differences in the risk factors appear to be indistinguishable.

Care is needed to prevent DM, and more efforts will be needed to reduce the risk of $\mathrm{DM}$ and the number of diabetic patients. In addition, more epidemiological studies in DM are needed to identify and alleviate the disease burden of DM and its complications. 
This study had some limitations. First, the HEALS and NHIS databases are secondary databases that were not planned and collected according to specific research objectives, thereby limiting the validity of variables' definitions and research results. These secondary data used limited by the lack of detailed clinical information to study a specific disease due to the data used for insurance claims. For example, the German Diabetes Association and the German Clinical Chemistry Association, in their 2019 guidelines, recommend caution using $\mathrm{HbA} 1 \mathrm{c}$ to diagnose diabetes in the elderly [41]. Nevertheless, it is difficult to discuss these issues in the data used. It may be that Korean doctors diagnosed and entered the diagnosis code according to the diabetes diagnostic criteria recommended by the latest diabetes guidelines. On the other hand, the use of secondary databases can include largescale groups of research participants, which makes it easier to generalize the results and reduces selective reporting. Second, this studyexcluded subjects without information on smoking, drinking, and exercise in the subject selection process. Such exclusion was an inevitable choice because health behavior information is important for finding the risk factors for the conversion to DM. Third, the changes in health behaviors during the followup period could not be analyzed due to data limitations. Despite these limitations, these findings provide comprehensive epidemiological information on diagnosed IFG and DM in the ROK using a large national population-based sample.

\section{Conclusions}

This epidemiological study in the ROK, which was a seven-year follow-up of nondiabetics from National Health Examinees, showed that the risk factors are similar to those for other regions and races. The incidence of progression to DM was 22.8 per 1000 person-years, which is higher than that found in previous studies in the ROK, but much lower than the incidence reported in an Asian Indian population.

Author Contributions: Conceptualization, M.K.H., J.H.P., K.H.K., S.-K.A. and S.M.J.; methodology, M.K.H., J.H.P., K.H.K., S.-K.A. and S.M.J.; formal analysis, J.H.P., K.H.K. and S.M.J.; writing-original draft preparation, M.K.H. and S.M.J.; writing-review and editing, M.K.H., J.H.P., K.H.K., S.-K.A. and S.M.J. All authors have read and agreed to the published version of the manuscript.

Funding: This research received no external funding.

Institutional Review Board Statement: This study was approved by the Institutional Review Board of Dongguk University, Gyeongju (DGU20150010).

Informed Consent Statement: Patient consent was exempted because of the total anonymity of all research data used in this study. The authors were able to analyze these data using a secure computer in the security room of NHIS.

Data Availability Statement: The dataset may not be taken out of the NHIS according to the policy of the National Health Insurance Service of Korea. The data can be accessed on the National Health Insurance Data Sharing Service homepage of the NHIS (https://nhiss.nhis.or.kr/bd/ab/bdaba000 eng.do (accessed on 27 October 2021)). Applications to use the NHIS-HEALS data will be reviewed by the inquiry committee of research support. Once approved, raw data will be provided to the applicant for a fee. Although the datasets are coded in English and numbers, not in Korean (Hangul), the use of individual data is allowed only for Korean researchers. Nevertheless, it would be possible for researchers outside the country to gain access to the data by conducting a joint study with Korean researchers.

Conflicts of Interest: The authors have no conflict of interest to declare with respect to the authorship and/or publication of this article.

\section{References}

1. Saeedi, P.; Petersohn, I.; Salpea, P.; Malanda, B.; Karuranga, S.; Unwin, N.; Colagiuri, S.; Guariguata, L.; Motala, A.A.; Ogurtsova, K. Global and regional diabetes prevalence estimates for 2019 and projections for 2030 and 2045: Results from the International Diabetes Federation Diabetes Atlas. Diabetes Res. Clin. Pract. 2019, 157, 107843. [CrossRef]

2. Zand, A.; Ibrahim, K.; Patham, B. Prediabetes: Why Should We Care? Methodist Debakey Cardiovasc. J. 2018, 14, 289-297. [CrossRef] [PubMed] 
3. ADA, 2. Classification and Diagnosis of Diabetes: Standards of Medical Care in Diabetes-2020. Diabetes Care 2020, 43, S14-S31.

4. ADA, 3. Prevention or Delay of Type 2 Diabetes: Standards of Medical Care in Diabetes-2020. Diabetes Care 2020, 43, S32-S36.

5. Tabák, A.G.; Herder, C.; Rathmann, W.; Brunner, E.J.; Kivimäki, M. Prediabetes: A high-risk state for developing diabetes. Lancet 2012, 379, 2279. [CrossRef]

6. Azami, M.; Sharifi, A.; Norozi, S.; Mansouri, A.; Sayehmiri, K. Prevalence of diabetes, impaired fasting glucose and impaired glucose tolerance in patients with thalassemia major in Iran: A meta-analysis study. Casp. J. Intern. Med. $2017,8,1$.

7. Andes, L.J.; Cheng, Y.J.; Rolka, D.B.; Gregg, E.W.; Imperatore, G. Prevalence of prediabetes among adolescents and young adults in the United States, 2005-2016. JAMA Pediatrics 2020, 174, e194498. [CrossRef]

8. Guerrero-Romero, F.; Violante, R.; Rodríguez-Morán, M. Distribution of fasting plasma glucose and prevalence of impaired fasting glucose, impaired glucose tolerance and type 2 diabetes in the Mexican paediatric population. Paediatr. Perinat. Epidemiol. 2009, 23, 363-369. [CrossRef]

9. Ma, R.C.; Chan, J.C. Type 2 diabetes in East Asians: Similarities and differences with populations in Europe and the United States. Ann. N. Y. Acad. Sci. 2013, 1281, 64-91. [CrossRef]

10. Anjana, R.M.; Shanthi Rani, C.S.; Deepa, M.; Pradeepa, R.; Sudha, V.; Divya Nair, H.; Lakshmipriya, N.; Subhashini, S.; Binu, V.S.; Unnikrishnan, R.; et al. Incidence of Diabetes and Prediabetes and Predictors of Progression Among Asian Indians: 10-Year Follow-up of the Chennai Urban Rural Epidemiology Study (CURES). Diabetes Care 2015, 38, 1441-1448. [CrossRef]

11. Tillin, T.; Hughes, A.D.; Wang, Q.; Wurtz, P.; Ala-Korpela, M.; Sattar, N.; Forouhi, N.G.; Godsland, I.F.; Eastwood, S.V.; McKeigue, P.M.; et al. Diabetes risk and amino acid profiles: Cross-sectional and prospective analyses of ethnicity, amino acids and diabetes in a South Asian and European cohort from the SABRE (Southall And Brent REvisited) Study. Diabetologia 2015, 58, 968-979. [CrossRef] [PubMed]

12. Kurotani, K.; Miyamoto, T.; Kochi, T.; Eguchi, M.; Imai, T.; Nishihara, A.; Tomita, K.; Uehara, A.; Yamamoto, M.; Murakami, T.; et al. Metabolic syndrome components and diabetes incidence according to the presence or absence of impaired fasting glucose: The Japan Epidemiology Collaboration on Occupational Health Study. J. Epidemiol. 2017, 27, 408-412. [CrossRef]

13. Lee, J.; Lee, J.S.; Park, S.-H.; Shin, S.A.; Kim, K. Cohort Profile: The National Health Insurance Service-National Sample Cohort (NHIS-NSC), South Korea. Int. J. Epidemiol. 2016, 46, e15. [CrossRef] [PubMed]

14. Seong, S.C.; Kim, Y.Y.; Park, S.K.; Khang, Y.H.; Kim, H.C.; Park, J.H.; Kang, H.J.; Do, C.H.; Song, J.S.; Lee, E.J.; et al. Cohort profile: The National Health Insurance Service-National Health Screening Cohort (NHIS-HEALS) in Korea. BMJ Open 2017, 7, e016640. [CrossRef]

15. Hur, K.Y.; Moon, M.K.; Park, J.S.; Kim, S.K.; Lee, S.H.; Yun, J.S.; Baek, J.H.; Noh, J.; Lee, B.W.; Oh, T.J.; et al. 2021 Clinical Practice Guidelines for Diabetes Mellitus of the Korean Diabetes Association. Diabetes Metab. J. 2021, 45, 461-481. [CrossRef] [PubMed]

16. Jeon, Y.; Son, K.Y. Effects of different definitions of low muscle mass on its association with metabolic syndrome in older adults: A Korean nationwide study. Geriatr. Gerontol. Int. 2021, 21, 1003-1009. [CrossRef]

17. Statistics Korea. Korean Statistical Information Service. Available online: https://kosis.kr/eng (accessed on 27 October 2021).

18. Lee, S.; Kim, J.-S.; Jung, J.-G.; Oh, M.-K.; Chung, T.-H.; Kim, J. Korean alcohol guidelines for moderate drinking based on facial flushing. Korean J. Fam. Med. 2019, 40, 204-211. [CrossRef]

19. Kim, K.S.; Park, S.W. Exercise and type 2 diabetes: ACSM and ADA joint position statement. J. Korean Diabetes 2012, 13, 61-68 [CrossRef]

20. Colberg, S.R.; Sigal, R.J.; Fernhall, B.; Regensteiner, J.G.; Blissmer, B.J.; Rubin, R.R.; Chasan-Taber, L.; Albright, A.L.; Braun, B. Exercise and type 2 diabetes: The American College of Sports Medicine and the American Diabetes Association: Joint position statement. Diabetes Care 2010, 33, e147-e167. [CrossRef]

21. Bae, N.-K.; Kim, K.-H.; Kwon, I.-S.; Cho, Y.-C. Changes in Prevalence of Obesity for 10 Years (1997 2007) and Its Related Factors in Health Checkup Examinees. J. Korea Acad. Ind. Coop. Soc. 2009, 10, 1091-1099.

22. Han, S.J.; Kim, H.J.; Kim, D.J.; Lee, K.W.; Cho, N.H. Incidence and predictors of type 2 diabetes among Koreans: A 12-year follow up of the Korean Genome and Epidemiology Study. Diabetes Res. Clin. Pract. 2017, 123, 173-180. [CrossRef] [PubMed]

23. HIRA Statistics about Diabetes. Available online: http:/ / opendata.hira.or.kr/op/opc/olapMfrnIntrsIlnsInfo.do (accessed on 27 October 2021).

24. Quan, J.; Li, T.K.; Pang, H.; Choi, C.H.; Siu, S.C.; Tang, S.Y.; Wat, N.M.S.; Woo, J.; Johnston, J.M.; Leung, G.M. Diabetes incidence and prevalence in Hong Kong, China during 2006-2014. Diabet. Med. A J. Br. Diabet. Assoc. 2017, 34, 902-908. [CrossRef] [PubMed]

25. Goto, A.; Goto, M.; Noda, M.; Tsugane, S. Incidence of type 2 diabetes in Japan: A systematic review and meta-analysis. PLoS ONE 2013, 8, e74699. [CrossRef]

26. Thomas, A.; Ashcraft, A. Type 2 Diabetes Risk among Asian Indians in the US: A Pilot Study. Nurs. Res. Pract. 2013, 2013, 492893. [CrossRef]

27. Selph, S.; Dana, T.; Bougatsos, C.; Blazina, I.; Patel, H.; Chou, R. Screening for Type 2 Diabetes Mellitus: A Systematic Review for the U.S. Preventive Services Task Force. Ann. Intern. Med. 2015, 162, 765-776. [CrossRef] [PubMed]

28. Akintunde, A.A. Epidemiology of conventional cardiovascular risk factors among hypertensive subjects with normal and impaired fasting glucose. S. Afr. Med. J. 2010, 100, 594-597. [CrossRef] [PubMed]

29. NICE. Preventing Type 2 Diabetes Overview; National Institute for Health and Care Excellence: London, UK, 2021. 
30. Ramachandran, A.; Snehalatha, C.; Satyavani, K.; Sivasankari, S.; Vijay, V. Metabolic syndrome does not increase the risk of conversion of impaired glucose tolerance to diabetes in Asian Indians-Result of Indian diabetes prevention programme. Diabetes Res. Clin. Pract. 2007, 76, 215-218. [CrossRef]

31. Maddatu, J.; Anderson-Baucum, E.; Evans-Molina, C. Smoking and the risk of type 2 diabetes. Transl. Res. 2017, $184,101-107$. [CrossRef]

32. Śliwińska-Mossoń, M.; Milnerowicz, H. The impact of smoking on the development of diabetes and its complications. Diab. Vasc. Dis. Res. 2017, 14, 265-276. [CrossRef]

33. Akter, S.; Goto, A.; Mizoue, T. Smoking and the risk of type 2 diabetes in Japan: A systematic review and meta-analysis. J. Epidemiol. 2017, 27, 553-561. [CrossRef]

34. Kim, J.H.; Noh, J.; Choi, J.W.; Park, E.C. Association of Education and Smoking Status on Risk of Diabetes Mellitus: A PopulationBased Nationwide Cross-Sectional Study. Int. J. Env. Res. Public. Health 2017, 14, 655. [CrossRef]

35. Cai, X.; Chen, Y.; Yang, W.; Gao, X.; Han, X.; Ji, L. The association of smoking and risk of diabetic retinopathy in patients with type 1 and type 2 diabetes: A meta-analysis. Endocrine 2018, 62, 299-306. [CrossRef] [PubMed]

36. Pan, A.; Wang, Y.; Talaei, M.; Hu, F.B. Relation of Smoking With Total Mortality and Cardiovascular Events Among Patients With Diabetes Mellitus: A Meta-Analysis and Systematic Review. Circulation 2015, 132, 1795-1804. [CrossRef] [PubMed]

37. Li, X.H.; Yu, F.F.; Zhou, Y.H.; He, J. Association between alcohol consumption and the risk of incident type 2 diabetes: A systematic review and dose-response meta-analysis. Am. J. Clin. Nutr. 2016, 103, 818-829. [CrossRef] [PubMed]

38. Aune, D.; Norat, T.; Leitzmann, M.; Tonstad, S.; Vatten, L.J. Physical activity and the risk of type 2 diabetes: A systematic review and dose-response meta-analysis. Eur. J. Epidemiol. 2015, 30, 529-542. [CrossRef]

39. Jenkins, D.W.; Jenks, A. Exercise and Diabetes: A Narrative Review. J. Foot. Ankle Surg 2017, 56, 968-974. [CrossRef]

40. Wahid, A.; Manek, N.; Nichols, M.; Kelly, P.; Foster, C.; Webster, P.; Kaur, A.; Friedemann Smith, C.; Wilkins, E.; Rayner, M.; et al. Quantifying the Association Between Physical Activity and Cardiovascular Disease and Diabetes: A Systematic Review and Meta-Analysis. J. Am. Heart Assoc. 2016, 5, e002495. [CrossRef]

41. Groos, S.; Kretschmann, J.; Macare, C.; Weber, A.; Hagen, B. Qualitätssicherungsbericht 2017 Disease Management Programme in Nordrhein: Tabellenband, Version 1c; Zentralinstitut für die kassenärztliche Versorgung in Deutschland: Berlin, Germany, 2018. 